Disponível em

http://www.anpad.org.br/rac

RAC, Rio de Janeiro, v. 19, n. 1, art. 4, pp. 65-83, Jan./Fev. 2015

http://dx.doi.org/10.1590/1982-7849rac20151779

$($ (c) EY-NO

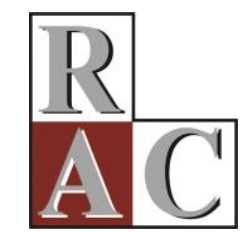

\title{
A Regulamentação Ambiental Conduzindo Estratégias Ecoinovativas na Indústria de Papel e Celulose
}

The Environmental Regulation Driving Eco-innovative Strategies in the Pulp and Paper Industry

Marlete Beatriz Maçaneiro Universidade Estadual do Centro-Oeste - UNICENTRO

Sieglinde Kindl da Cunha Universidade Positivo - UP; Universidade Federal do Paraná - UFPR

Marcos Roberto Kuhl Universidade Estadual do Centro-Oeste - UNICENTRO

João Carlos da Cunha Universidade Positivo - UP

Artigo recebido em 10.12.2013. Última versão recebida em 19.08.2014. Aprovado em 24.08.2014. 


\title{
Resumo
}

Objetivou-se analisar, neste estudo, a associação da regulamentação ambiental com a adoção de estratégias de ecoinovação proativas e reativas na indústria brasileira de celulose, papel e produtos de papel. A metodologia foi baseada na abordagem quantitativa, utilizando a estratégia de levantamento de corte transversal, por meio de um questionário on-line autoadministrado, respondido por 117 empresas do setor, de todos os portes e de todas as regiões do Brasil. Para a análise dos dados, foi utilizada a estatística inferencial pela análise de regressão logística. Os principais resultados apontam que as organizações participantes deste estudo consideram a regulamentação ambiental como condutora de estratégias de ecoinovação proativas, de forma mais contundente que as reativas, em oposição à literatura anterior. Este resultado se insere na perspectiva evolucionista, a qual postula que as empresas veem a regulamentação ambiental menos como custo e mais como oportunidade, orientando-as para melhoria de produtividade e competitividade.

Palavras-chave: regulamentação ambiental; ecoinovação; estratégias reativas e proativas; indústria de papel e celulose.

\begin{abstract}
This study aimed to analyze the association of environmental regulation with the adoption of both proactive and reactive eco-innovation strategies in the Brazilian pulp, paper, and paper product industry. Methodology was based on a quantitative approach, using a cross-sectional survey by means of a self-administered online questionnaire answered by 117 sector companies of all sizes and Brazilian regions. Data analysis consisted of inferential statistics with logistic regression analysis. The main results show that the organizations participating in this study consider environmental regulation as conducive of proactive, more than reactive, eco-innovation strategies, as opposed to previous literature. This result fits into the evolutionary perspective, which postulates that companies see environmental regulation as less a cost and more an opportunity, directing them to improve productivity and competitiveness.
\end{abstract}

Key words: environmental regulations; eco-innovation; reactive and proactive strategies; pulp and paper industry. 


\section{Introdução}

O tema da sustentabilidade ambiental tem sido debatido no âmbito acadêmico, nas últimas décadas, em seus vários aspectos e em diversas áreas do conhecimento, tratando das preocupações com o meio ambiente. Todavia a grande parte dos estudos na área de inovação tem se mantido estreitamente ligada a preocupações de ordem econômica, como competitividade, pressões da demanda, entre outras. Ambos os temas têm encontrado dificuldades em incorporar os seus processos inerentes de forma a tratar a gestão ambiental no contexto da inovação, assim como a gestão da inovação amparada nos pressupostos da área ambiental (Andrade, 2004).

Estudiosos da Teoria Econômica da Inovação, como Freeman (1996), porém, têm tratado também das questões ambientais. E Freeman (1996) alerta que, com o aumento da concentração sobre o "efeito estufa", mais atenção tem sido dada à mudança institucional (incentivos econômicos e sanções) e menor atenção à mudança técnica. Menciona, o autor, ainda, que a reversão da maioria dos riscos ao meio ambiente depende não só dos métodos de regulamentação, de incentivos econômicos e de outras mudanças institucionais, mas também de contínua mudança tecnológica. Algumas inovações técnicas com fontes renováveis de energia podem fazer grande diferença às perspectivas futuras.

Estudos nessa linha tratam das ecoinovações, que são definidas como inovações com ênfase no desenvolvimento sustentável. Neste conceito, o processo produtivo resulta, em todo o seu ciclo de vida, na redução dos encargos ambientais, com isso, contribuindo para melhorar o desenvolvimento sustentável (Rennings, 2000). O conceito de ecoinovação é relativamente novo, oriundo das recentes discussões e preocupações com os impactos ambientais. O termo ecoinovação, propriamente dito, foi utilizado, pela primeira vez, por Fussler e James, em seu livro Driving Eco-Innovation: A Breakthrough Discipline for Innovation and Sustainability, publicado em 1996.

Rennings (2000) considera que "as ecoinovações podem ser desenvolvidas por empresas ou organizações sem fins lucrativos, podem ser negociadas em mercados ou não, sua natureza pode ser tecnológica, organizacional, social ou institucional" (p. 322). As tecnológicas podem ser distinguidas em curativas e preventivas, sendo que as primeiras reparam danos ao meio ambiente e são denominadas de soluções end-of-pipe; enquanto que as preventivas tentam evitá-los e são as denominadas soluções cleaner production, que fazem parte de uma abordagem mais ampla denominada prevenção da poluição (Rennings, 2000).

De acordo com Buysse e Verbeke (2003), Hart (1995) e Rennings (2000), as tecnologias end-ofpipe refletem uma postura de controle da poluição no final do processo produtivo, sendo reativas à regulamentação ambiental. Normalmente, são dispositivos que reparam danos e demandam caros investimentos de capital para instalação e operação. Esses mesmos autores mencionam que, em contraste, as soluções cleaner production implicam na utilização de métodos de melhoria contínua durante o processo de produção. São focalizadas em objetivos ambientais bem definidos a fim de reduzir os níveis de poluição abaixo dos requisitos legais. Essas soluções preventivas resultam em aumento da produtividade e eficiência da organização, uma vez que haverá redução de custos por conta de menos resíduos resultantes, ciclos mais curtos de produção e melhor utilização dos recursos e capacidades (Buysse \& Verbeke, 2003; Hart, 1995; Rennings, 2000).

As ecoinovações organizacionais são aquelas mudanças nos "instrumentos de gestão ao nível da empresa como ecoauditorias, que são de importância crescente para a inovação" (Rennings, 2000, p. 323). As ecoinovações sociais são refletidas nos padrões de consumo sustentáveis, as quais têm recebido atenção crescente, sendo consideradas como mudanças nos valores das pessoas e seus estilos de vida para a sustentabilidade. Por fim, as institucionais se referem às respostas institucionais inovadoras aos problemas de sustentabilidade, tais como as redes locais e agências, governança global e comércio internacional (Rennings, 2000).

Estudos sobre este tema apontam para o fato de haver relativamente poucas pesquisas e ações que trabalhem a interseção entre a inovação e a sustentabilidade ambiental, resultando em incertezas 
teóricas, metodológicas e políticas para implementações e gestões nesse sentido (Andersen, 2010; Andrade, 2004; Maçaneiro, Cunha, \& Balbinot, 2013). Tal fato caracteriza essa área como um campo ainda pouco explorado, mas que vem ganhando crescente atenção na literatura internacional, de forma mais presente no âmbito dos países da União Europeia e Estados Unidos.

A ecoinovação é incentivada por vários fatores externos às organizações, incluindo principalmente a regulamentação ambiental. Autores como Ashford (2000) e Porter e Linde (1995) ressaltam que os regulamentos devem orientar a empresa a inovar na área ambiental, e a empresa deve ver esta pressão como melhoria de produtividade para a competitividade. As questões do meio ambiente devem ser vistas, pelas empresas, como um estímulo à geração de inovações e oportunidades tecnológicas, econômicas e competitivas, sendo consideradas estratégias proativas (Ashford, 2000). Ou seja, o conhecimento gerencial e as atitudes em relação às mudanças tecnológicas e às preocupações com o meio ambiente devem ser estimulados pela regulamentação ambiental (Ansanelli, 2011; Ashford, 2000; Porter \& Linde, 1995).

Nesse sentido, as empresas adotam estratégias em diferentes amplitudes para as questões relacionadas ao meio ambiente, tais como as reativas e as proativas. Sharma (2000) e Sharma, Pablo e Vredenburg (1999) definem estratégias reativas como os resultados na forma de ações para o cumprimento regulamentar; em outras palavras, são as ações impostas externamente pela legislação ambiental. Essas estratégias não passam de cumprimento da legislação através de controle da poluição, com investimentos em tecnologias corretivas para remediar os problemas no final do processo produtivo (end-of-pipe) (Buysse \& Verbeke, 2003). Por isso, essas estratégias não requerem que a empresa desenvolva competências ou habilidades na produção de novas tecnologias ou novos processos ambientais (Sharma, 2000).

Ao contrário, as estratégias proativas são aquelas ações voluntárias de redução maior dos impactos ambientais das operações, dessa forma, criando vantagem competitiva por meio da adoção de tecnologias ecoinovadoras (Sharma, Pablo, \& Vredenburg, 1999). Essas inovações são definidas como estratégias ambientais de prevenção da poluição ou voluntaristas, que exigem a aquisição e instalação de novas tecnologias, envolvendo aprendizagem maior e desenvolvendo capacidades organizacionais competitivas (Buysse \& Verbeke, 2003; Sharma et al., 1999). Neste posicionamento estratégico, os atores levam em consideração as questões ambientais como oportunidade de ganhos concorrenciais (Aragón-Correa, 1998; Sharma et al., 1999). No entanto, Sharma, Aragón-Correa e Rueda-Manzanares (2007) ressaltam que, "mesmo com a evidência acumulada na última década que as estratégias ambientais proativas tendem a ser acompanhadas por melhor desempenho financeiro, ainda não temos um entendimento bem desenvolvido de por que apenas algumas empresas em uma indústria implementam essas estratégias" (p. 269).

Portanto são necessárias pesquisas que tratem de fatores que conduzam à realização de ações ambientalmente favoráveis, tais como a regulamentação ambiental. Para verificar essas questões, optouse por realizar esta pesquisa em empresas do setor de celulose, papel e produtos de papel do Brasil. A escolha desse setor é justificada por suas atividades serem consideradas de alto potencial de poluição e devido ao uso de recursos naturais, conforme consta na lei que aprovou a Política Nacional do Meio Ambiente brasileira (Lei n. 6.938, 1981), fazendo com que seja um importante objeto de estudo, além da sua relevância para o desenvolvimento do país.

Nesse sentido, este estudo se orienta a partir do seguinte problema de pesquisa: as empresas industriais do setor de celulose, papel e produtos de papel consideram a regulamentação ambiental como custo (ameaça) ou oportunidade estratégica, resultando em ações reativas ou proativas? A partir desse questionamento, definiu-se o objetivo de analisar a associação da regulamentação ambiental com a adoção de estratégias de ecoinovação proativas e reativas no setor brasileiro de celulose, papel e produtos de papel.

Assim, este estudo está centrado na importância de analisar as estratégias de ecoinovação e o fator regulamentação que compõe um dos condutores, no sentido de verificar a sua relevância na definição das estratégias organizacionais. Para tanto, foram definidos dois construtos, um denominado de 
regulamentação ambiental, composto por quatro variáveis independentes, e outro definido como estratégias de ecoinovação, composto por 13 variáveis dependentes. Com isso, buscou-se analisar as associações entre as variáveis e testar as hipóteses que serão apresentadas no próximo tópico.

\section{Referencial Teórico e Definição das Hipóteses do Estudo}

De acordo com Foxon e Andersen (2009), a criação de política de inovação tratou muito pouco com a área ambiental até recentemente, sendo que as questões ambientais têm sido amplamente vistas como um custo para as empresas. Acrescentam, esses autores, que o desenvolvimento dos dois tipos de políticas, ambiental e de inovação, deve caminhar de forma paralela e com pequenas interações. Rennings (2000) também ressalta que as duas políticas devem ser vistas de forma complementar, em que a de ecoinovação pode ajudar a reduzir os custos da inovação social, institucional e tecnológica, especialmente nas fases de invenção e introdução no mercado; e, na fase de difusão, pode ajudar a melhorar as características de desempenho dos produtos/tecnologias.

Corazza (2003a) complementa que os dois tipos de políticas devem influenciar a criação de tecnologias, direcionando e controlando essa escolha. Não apenas isso, mas também essas políticas devem avaliar o desempenho e o impacto das tecnologias para que se tenha uma situação desejável em termos de proteção ambiental.

Em vista disso, é de fundamental importância o quadro regulatório e de política ambiental como fator determinante para o comportamento ecoinovativo nas empresas e instituições. Foray e Grübler (1996) mencionam que, durante os anos de 1970, "as políticas ambientais foram amplamente direcionadas para a difusão forçada de uma série de tecnologias limpas 'end-of-pipe' incrementais" (p. 4). No entanto são necessárias mudanças radicais nas regulamentações, o que sugere uma nova lógica para a política, que é complexa, porque requer complementariedade entre o apoio para geração de alternativas tecnológicas de longo prazo e políticas de controle da poluição ambiental no curto prazo. Esses autores ainda ressaltam que não só é necessária a mudança nas políticas, mas também novas estratégias empresariais que favoreçam a mudança tecnológica e organizacional incremental e radical (Foray \& Grübler, 1996).

Nesse sentido, Freeman (1996) defende que os novos mecanismos regulatórios devem garantir a difusão das tecnologias de energia limpa e renovável e de dispositivos de conservação de materiais. Mas afirma que isso é questão de difícil resolução, pois depende da combinação de políticas sociais, econômicas, da política de ciência e tecnologia e de um sistema de regulação legal. Ainda, Andrade (2004) ressalta que a construção de políticas tecnológicas inovadoras e ambientais deve abandonar a postura defensiva e restritiva, com tal atitude, partindo para mecanismos que permitam a construção de processos inovativos voltados à sustentabilidade.

De acordo com Schmidheiny (1992), os mecanismos de regulação ambiental existentes são de três tipos: (a) mecanismos de comando e controle, que são as regulamentações governamentais que impõem padrões ambientais no trato dos recursos naturais; (b) mecanismos de autorregulação, com a adoção de padrões ambientais pelas empresas ou setores industriais; (c) instrumentos econômicos imputados por ação governamental de alteração de preços de recursos, afetando a produção e o consumo. Em muitos países, os instrumentos econômicos estão sendo usados de forma crescente, como mecanismos para melhorar o desempenho da gestão ambiental. Já os regulamentos do tipo comando e controle são aqueles que incluem multas ou sanções para as degradações ambientais (Motta \& Young, 1997).

Ashford (2000) menciona que algum grau de incerteza sobre a futura regulamentação é benéfico para a inovação e que o rigor regulatório é também um fator importante para a definição de inovação tecnológica por parte das empresas. Todavia, para Foray e Grübler (1996), a regulamentação pode criar um novo risco à inovação, além dos inerentes ao processo inovativo, no qual não se tem como prever se 
ela cumprirá as normas ambientais atuais e futuras. Esse risco é o da incerteza sobre se a regulamentação futura trará ou não restrições mais severas quanto às inovações atuais, o que se torna um desincentivo aos inovadores para alocarem recursos e investimentos na P\&D ecoinovativa (Foray \& Grübler, 1996).

No entanto há um conjunto substancial de literatura que segue a linha de Porter, que considera o rigor regulatório e a antecipação de regulamentação importantes propulsores de inovação. Porter e Linde $(1995,1999)$ cunharam a chamada hipótese de Porter, a qual postula que, se a regulamentação for devidamente formulada e as empresas estiverem sintonizadas com as possibilidades de ganhos, a inovação pode minimizar e até mesmo compensar o custo de conformidade. Ou seja, uma regulamentação ambiental mais rigorosa e específica pode forçar as empresas poluentes a buscarem inovações para reduzir o custo da melhoria do impacto ambiental, incrementar também a competitividade e levar a uma relação positiva entre o desempenho ambiental e econômico.

Porter e Linde (1995) mencionam ainda que, em resposta à regulamentação ambiental, a inovação pode assumir duas formas: uma que aumenta o conhecimento das empresas sobre como lidar com a poluição; e a outra, ao mesmo tempo em que aborda os impactos ambientais, melhora o próprio produto afetado e/ou processos relacionados. Essa última forma é a que os autores consideram como o tipo de inovação fundamental para a afirmação de que a regulamentação ambiental pode realmente aumentar a competitividade industrial. Para tanto, acredita-se que a regulamentação tem influência importante na direção da inovação, seja de forma positiva ou negativa (Porter \& Linde, 1995).

Não obstante às questões até aqui mencionadas, a literatura também considera que os programas de sustentabilidade ambiental normalmente não são vistos como importantes pelos executivos e não fazem parte das questões essenciais da empresa (Blackburn, 2008). A busca da sustentabilidade pelas organizações, muitas vezes, é em resposta a problemas com ativistas, com a mídia e, principalmente, com a regulamentação ambiental. Nidumolu, Prahalad e Rangaswami (2009) ressaltam que há a noção de que as empresas acreditam que quanto mais amigáveis se tornam do meio ambiente, mais aumentam os custos, que há perda de produtividade e não há benefícios de curto prazo.

As empresas consideram que os custos adicionais são elevados para o trato das questões ambientais, as quais são tidas como ameaças à sua sobrevivência, reduzindo a competitividade (Romeiro \& Salles, 1996; Young, Podcameni, Mac-Knight, \& Oliveira, 2009). Em muitos casos, essa questão não é tratada como estratégia proativa de negócios, e sim são tomadas ações, estratégias reativas.

No caso brasileiro, de acordo com Motta e Young (1997), as políticas governamentais de gestão ambiental são mais relacionadas aos instrumentos econômicos (royalties, compensações fiscais, cobranças ao usuário e tributação florestal). Essa tendência é enfatizada pelo interesse na geração de receitas para o setor da gestão ambiental nacional. Além disso, esses autores destacam que, no decorrer dos anos, as falhas de aplicação da legislação criaram um descrédito na política ambiental brasileira e aumentaram os custos burocráticos e incertezas.

Isso faz com que as organizações percebam as exigências da legislação ambiental como um fator de aumento de custos e um risco à sua produtividade (Young et al., 2009). Essas questões tornam-se um obstáculo jurídico-legal e demandam grandes investimentos, além de elas não considerarem a possibilidade de recuperação desses investimentos. Por sua vez, a indústria tem sofrido crescentes pressões para melhorar o desempenho ambiental, oriundas dos órgãos reguladores, entre outros fatores (Barbieri, 2011; Donaire, 2007).

Corazza (2003b), todavia, menciona que até a década de 1980 havia essa perspectiva reativa da internalização dos custos ambientais do processo de produção, imputados pela regulamentação. No entanto a autora afirma que, a partir dos anos de 1980, esta visão começou a ser rompida, tanto na prática das organizações quanto nas suas análises, então, focalizando para motivações proativas dessa internalização.

Assim, as questões do meio ambiente devem ser vistas pelas empresas como um estímulo à geração de inovações e oportunidades tecnológicas, econômicas e competitivas (Ansanelli, 2011), sendo consideradas como estratégias proativas. O conhecimento gerencial e as atitudes em relação às mudanças 
tecnológicas e às preocupações com o meio ambiente devem ser estimulados pela regulamentação ambiental (Ashford, 2000). Ou seja, a regulamentação deve orientar a empresa a inovar, e a empresa deve ver esta pressão como melhoria de produtividade para a competitividade (Porter \& Linde, 1995).

Portanto é necessário analisar as estratégias de ecoinovação das empresas industriais brasileiras, com foco na regulamentação ambiental, se elas consideram um custo (ameaça) ou oportunidade estratégica, resultando em ações reativas ou proativas. Nesse contexto, levando-se em consideração a literatura mencionada, foram levantadas duas hipóteses, desdobradas em uma hipótese nula e uma alternativa para cada uma delas, nos seguintes termos:

$\mathbf{H}_{0} \mathrm{~A}$ : A percepção dos executivos sobre a regulamentação ambiental é que ela está associada positivamente à adoção de estratégias de ecoinovação reativas.

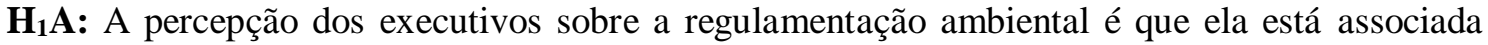
negativamente à adoção de estratégias de ecoinovação reativas.

$\mathbf{H}_{0}$ B: A percepção dos executivos sobre a regulamentação ambiental é que ela está associada positivamente à adoção de estratégias de ecoinovação proativas.

H1B: A percepção dos executivos sobre a regulamentação ambiental é que ela está associada negativamente à adoção de estratégias de ecoinovação proativas.

Todavia as consequências da gestão ou não da ecoinovação significam posições na concorrência e a própria permanência ou saída do mercado. Na atualidade, em meio aos aspectos ligados à gestão da ecoinovação, as empresas deveriam passar a considerar a proteção ambiental não mais como uma exigência punida com multas e sanções. Nesse novo cenário, essa questão passa a fazer parte das estratégias organizacionais de definição de ameaças e oportunidades (Donaire, 2007). Para tanto, são necessários processos organizacionais que demandem recursos diversos, assim como envolvam esforços significativos, por parte dos empresários, para tornar sustentáveis esses processos.

\section{Procedimentos Metodológicos e Composição dos Construtos do Estudo}

A metodologia deste estudo foi embasada na abordagem quantitativa e, para tanto, utilizou-se a estratégia de levantamento de corte transversal (survey cross-sectional). A pesquisa foi realizada com 117 empresas do setor de celulose, papel e produtos de papel, de todos os portes e de todas as regiões do Brasil, cujos dados foram coletados no período de julho a outubro de 2012. A caracterização da amostra será realizada no início do próximo tópico.

O instrumento utilizado para a coleta de dados foi o questionário computadorizado no formato autoadministrado, o qual foi enviado pela Internet, utilizando-se o sistema Qualtrics®. O questionário foi testado em termos de validade de conteúdo por três professores especialistas e também foi realizado um pré-teste, com três gerentes responsáveis pela área de gestão ambiental de empresas do setor e dois professores universitários da área de estratégia e sustentabilidade.

Para a mensuração, as questões de percepção dos respondentes foram elaboradas com emprego de escala balanceada de cinco pontos, com uma posição neutra. As escalas balanceadas são aquelas "com o mesmo número de categorias favoráveis e desfavoráveis" (Malhotra, 2006, p. 270). De acordo com Hair, Babin, Money e Samouel (2005), essas escalas podem ser consideradas como intervalares, as quais utilizam:

números para classificar objetos ou eventos de modo que a distância entre os números seja igual.... Quando os pesquisadores usam escalas intervalares em administração, tentam medir conceitos como atitudes, percepções, sentimentos, opiniões e valores através das chamadas escalas de classificação. (Hair, Babin, Money, \& Samouel, 2005, p. 184). 
Ainda, segundo Hair et al. (2005, p. 184), "tornou-se habitual na pesquisa em administração" a utilização de escala intervalar (normalmente utilizada nos estudos em administração, independentemente do número de pontos). Para esses autores, em termos restritos, são escalas ordinais, mas tratadas como se fossem intervalares. No entanto, segundo Hair, Black, Babin, Anderson e Tatham (2009, p. 25), "a única diferença real entre escalas intervalares e escalas de razão é que as primeiras têm um ponto zero arbitrário, enquanto as escalas de razão incluem um ponto zero absoluto". Assim, tratase de uma escala intervalar. Beuren e Oro (2014), Costa, Borini e Amatucci (2013), Kuhl e Cunha (2013) e Ribeiro, Oliveira e Borini (2012) são apenas alguns dos exemplos recentes da utilização de escalas intervalares em estudos quantitativos, com análises paramétricas, relacionados à inovação.

A população utilizada para envio do instrumento de coleta de dados foi constituída de 672 empresas de todo o território brasileiro, das quais se obteve acesso aos dados de e-mail e telefone. Os contatos foram inicialmente por e-mail, em que se explicaram os objetivos do estudo e a forma de respostas ao questionário. Posteriormente, foram realizados contatos, via telefone, com os responsáveis pela gestão ambiental nas empresas. Salienta-se que esforços significativos foram realizados para a obtenção de maior número de empresas para a análise deste estudo, com insistência de contatos, por telefone e e-mail, com as empresas. No entanto não se obteve sucesso maior nas respostas, apesar de ter sido estendida a coleta de dados por um período de quatro meses.

Foi verificado que, dessa população de empresas, 135 acessaram a página do sistema Qualtrics ${ }^{\circledR}$ para responder ao questionário, porém apenas 97 efetivamente concluíram todos os blocos do instrumento, inclusive com a identificação dessas empresas. Verificou-se também que outras 20 empresas não haviam se identificado, mas estavam com os demais dados completos para análise. Mas 18 empresas que iniciaram o preenchimento do questionário e não o concluíram, nem mesmo as respostas às variáveis, foram descartadas da análise. Portanto a quantidade final da amostra foi de 117 empresas. Nesse sentido, a amostragem da pesquisa foi definida como não probabilística (não aleatória), e sim por adesão, em que determinadas pessoas foram convidadas a responder ao questionário, mas poderiam decidir se iriam participar ou não da pesquisa (Cooper \& Schindler, 2011).

Antes da execução da análise dos dados, foi realizada a validação e limpeza dos dados no sentido de se eliminar possíveis falhas de distorções, resultantes de erros no preenchimento do instrumento, verificando-se valores ausentes nas respostas e outliers. Nesse sentido, foi realizada a análise de cada variável, por meio do gráfico Boxplot, e não foram detectados outliers entre as respostas dessas 117 empresas. Também não foram detectados valores faltantes no interior das questões (missing values), já que o sistema eletrônico utilizado não permitia que o respondente deixasse de marcar as opções de respostas.

A análise dos dados foi embasada na análise multivariada de dados, mais especificamente na Regressão Logística (RL). "A regressão logística é uma forma especializada de regressão que é formulada para prever e explicar uma variável categórica binária (dois grupos)" (Hair et al., 2009, p. 225). Para realização das análises, foi utilizado o pacote estatístico Statistical Package for the Social Sciences $\left(\mathrm{SPSS}^{\circledR}\right)$.

Essas análises ocorreram a partir das hipóteses apresentadas no tópico 2 e pelo construto de regulamentação ambiental, composto por quatro variáveis independentes; e um construto denominado estratégias de ecoinovação, composto por 13 variáveis dependentes. Esses fatores foram definidos em termos constitutivos e operacionais, conforme apresentados na sequência, com as variáveis construídas com base na literatura pesquisada.

Para o construto da regulamentação ambiental, considerou-se que são aqueles instrumentos definidos como normas jurídicas em relação ao desempenho ambiental, tais como os de comando e controle. Há também os instrumentos econômicos, que são aqueles que afetam os custos e o consumo, além da autorregulação por parte das empresas ou setores da indústria (Schmidheiny, 1992).

Para a mensuração desse construto, utilizaram-se quatro variáveis, conforme apresentadas na Tabela 1. Foi usada uma escala balanceada com cinco pontos e uma posição neutra, que variou entre grau de relevância muito pequeno a muito grande. O construto de regulamentação ambiental foi 
dividido em duas variáveis relacionadas com a regulamentação vista como custo/ameaça e outras duas relativas à regulamentação vista como oportunidade. Ressalta-se que a fundamentação teórica que embasou a definição dessas variáveis consta na fonte da tabela.

Tabela 1

Variáveis Empregadas para Mensurar o Construto Regulamentação Ambiental

Variável Questão: Nas opções abaixo, avalie o grau de relevância, no âmbito da empresa, dos regulamentos/legislações ambientais sobre cada uma das seguintes variáveis:

\begin{tabular}{|c|c|c|}
\hline \multirow{2}{*}{ 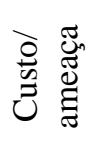 } & Var01 & Na aquisição de tecnologia de controle da poluição no final do processo produtivo. \\
\hline & Var02 & $\begin{array}{l}\text { No aumento de custo por sanções fiscais e/ou administrativas de responsabilidade por danos } \\
\text { ambientais, traduzindo-se em ameaça ao crescimento dos negócios. }\end{array}$ \\
\hline \multirow{2}{*}{ 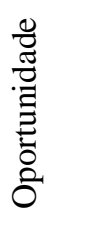 } & Var03 & $\begin{array}{l}\text { No desenvolvimento ou aquisição de novos produtos/processos/tecnologias inovadoras de } \\
\text { prevençãa da poluição, envolvendo aprendizagem contínua e desenvolvendo capacidades. } \\
\text { organizacionais. }\end{array}$ \\
\hline & Var04 & $\begin{array}{l}\text { A regulamentação serve como orientação para a empresa inovar, aprender e mudar suas } \\
\text { práticas, sendo que essa pressão é vista como melhoria de produtividade e competitividade. }\end{array}$ \\
\hline
\end{tabular}

Nota. Fonte: Elaborado pelos autores com base em Blackburn, W. R. (2008). The sustainability handbook. Washington: Environmental Law Institute; Buysse, K., \& Verbeke, A. (2003). Proactive environmental strategies: a stakeholder management perspective. Strategic Management Journal, 24(5), 453-470. doi: 10.1002/smj.299; Corazza, R. I. (2003b). Gestão ambiental e mudanças da estrutura organizacional. RAE-Eletrônica, 2(2), 1-23. Recuperado de http://www.scielo.br/pdf/raeel/v2n2/v2n2a06.pdf. doi: 10.1590/S1676-56482003000200006; Hart, S. (1995). A naturalresource-based view of the firm. The Academy of Management Review, 20(4), 986-1014. doi: 10.5465/AMR.1995.9512280033; Nidumolu, R., Prahalad, C. K., \& Rangaswami, M. R. (2009). Why sustainability is now the key driver of innovation. Harvard Business Review, 87(9), 56-64; Sharma, S. (2000). Managerial interpretations and organizational context as predictors of corporate choice of environmental strategy. Academy of Management Journal, 43(4), 681-697. doi: 10.2307/1556361; Sharma, S., Pablo, A. L., \& Vredenburg, H. (1999). Corporate environmental responsiveness strategies: the importance of issue interpretation and organizational context. The Journal of Applied Behavioral Science, 35(1), 87-108. doi: 10.1177/0021886399351008

O construto das estratégias de ecoinovação foi dividido em estratégias proativas e estratégias reativas. Para a definição constitutiva desse construto, levou-se em consideração que "as empresas com uma estratégia reativa atribuem grande importância à regulamentação do governo, mas apenas em um sentido estático, como uma resposta quase mecanicista e diária de rotina orientada para as novas exigências regulamentares" (Buysse \& Verbeke, 2003, p. 460). São processos caros, normalmente, improdutivos e que refletem uma postura reativa e seletiva em relação às questões ambientais, basicamente com tecnologias end-of-pipe.

Já as estratégias proativas são aquelas ações voluntárias de redução maior dos impactos ambientais das operações, as quais são apoiadas pela gestão superior da organização e criam vantagem competitiva por meio da adoção de tecnologias ambientais inovadoras (Menguc, Auh, \& Ozanne, 2010; Sharma, 2000; Sharma et al., 1999). São definidas como estratégias de prevenção da poluição ou voluntaristas, que exigem a aquisição e instalação de novas tecnologias, envolvendo aprendizagem contínua, desenvolvendo capacidades organizacionais competitivas e gerindo a qualidade total da organização (Buysse \& Verbeke, 2003; Hart, 1995; Sharma, 2000). Elas têm sido vistas a partir de uma perspectiva competitiva e o termo é usado para descrever atividades inovadoras de prevenção da poluição (Menguc, et al., 2010).

Neste estudo, operacionalmente, a tendência ao desenvolvimento de ações estratégicas de ecoinovações foi determinada por meio de variáveis, fundamentadas na literatura abordada, constantes da Tabela 2. Na questão, foram incluídas 13 variáveis, utilizando-se escala Likert de cinco pontos, que variou de discordo totalmente a concordo totalmente. A média das cinco primeiras variáveis compôs o construto estratégias reativas e a média das outras oito variáveis compôs o construto estratégias proativas. Neste caso, também, a fundamentação teórica que embasou a definição dessas variáveis como estratégias proativas e reativas consta na fonte da tabela. 
Tabela 2

\section{Variáveis Empregadas para Mensurar o Construto Estratégias de Ecoinovação}

\begin{tabular}{|c|c|c|}
\hline & Variável & $\begin{array}{l}\text { Questão: Nas opções abaixo, avalie o grau de desenvolvimento em sua empresa das atividades } \\
\text { relacionadas ao meio ambiente: }\end{array}$ \\
\hline \multirow{5}{*}{ 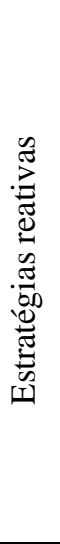 } & Var05 & $\begin{array}{l}\text { A empresa apenas se preocupa com a poluição no final do processo produtivo, por meio de } \\
\text { tecnologia de remediação, tais como a descontaminação do solo degradado. }\end{array}$ \\
\hline & Var06 & $\begin{array}{l}\text { A empresa apenas adquire tecnologias de controle de poluição (end-of-pipe), que objetiva tratar } \\
\text { a poluição antes que seja lançada ao meio ambiente, tais como: estações de tratamento de } \\
\text { efluentes, ciclones, precipitadores eletrostáticos, filtros, incineradores, etc. }\end{array}$ \\
\hline & Var07 & $\begin{array}{l}\text { A empresa investe em tecnologias e ações ambientais somente para o cumprimento da legislação } \\
\text { ambiental. }\end{array}$ \\
\hline & Var08 & $\begin{array}{l}\text { A empresa investe em tecnologias e ações ambientais somente como uma estratégia para } \\
\text { resolver problemas com ativistas e a mídia. }\end{array}$ \\
\hline & Var09 & $\begin{array}{l}\text { A empresa considera a gestão ambiental como um custo adicional, que pode prejudicar o } \\
\text { crescimento dos negócios. }\end{array}$ \\
\hline \multirow{8}{*}{ 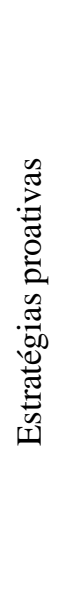 } & Var10 & A empresa usa recursos de marketing para tratar da gestão ambiental. \\
\hline & Var11 & $\begin{array}{l}\text { A empresa desenvolve ações ambientais no trabalho administrativo (reciclagem de papel, uso } \\
\text { de material reciclado, redução do uso de material, etc.). }\end{array}$ \\
\hline & Var12 & $\begin{array}{l}\text { A empresa desenvolve ações ambientais no trabalho produtivo (minimização de resíduos, uso } \\
\text { de energia renovável, reutilização de água, tratamento e eliminação segura de resíduos } \\
\text { perigosos, redução da produção de } \mathrm{CO} 2 \text {, reaproveitamento de matéria-prima, etc.). }\end{array}$ \\
\hline & Var13 & A empresa realiza auditorias ambientais periódicas. \\
\hline & Var14 & A empresa realiza a análise ambiental do ciclo de vida dos seus produtos. \\
\hline & Var15 & A empresa realiza parcerias/acordos com outras empresas/instituições para ações ambientais. \\
\hline & Var16 & A empresa possui ou viabiliza programas de formação ambiental para os gestores e funcionários. \\
\hline & Var17 & A empresa possui um sistema de prevenção de acidentes ambientais que possam ocorrer. \\
\hline
\end{tabular}

Nota. Fonte: Elaborado pelos autores com base em Aragón-Correa, J. A. (1998). Strategic proactivity and firm approach to the natural environment. Academy of Management Journal, 41(5), 556-567. doi: 10.2307/256942; Barbieri, J. C. (2011). Gestão ambiental empresarial: conceitos, modelos e instrumentos (3a ed.) São Paulo: Saraiva; Blackburn, W. R. (2008). The sustainability handbook. Washington: Environmental Law Institute; Buysse, K., \& Verbeke, A. (2003). Proactive environmental strategies: a stakeholder management perspective. Strategic Management Journal, 24(5), 453-470. doi: 10.1002/smj.299; Caracuel, J. A., Torres, M. Á. E., Torres, N. E. H., \& Salazar, M. D. V. (2011). La influencia de la diversificación y experiencia internacional en la estrategia medioambiental proactiva de las empresas. Investigaciones Europeas de Direccion y Economia de la Empresa, 17(1), 75-91. doi: 10.1016/S1135-2523(12)60045-8; Corazza, R. I. (2003b). Gestão ambiental e mudanças da estrutura organizacional. RAE-Eletrônica, 2(2), 1-23. Recuperado de http://www.scielo.br/pdf/raeel/v2n2/v2n2a06.pdf. doi: 10.1590/S1676-56482003000200006; Donaire, D. (2007). Gestão ambiental na empresa (2a ed.). São Paulo: Atlas; Foxon, T., \& Andersen, M. M. (2009, June). The greening of innovation systems for eco-innovation - towards an evolutionary climate mitigation policy. Proceedings of the DRUID Summer Conference - Innovation, Strategy and Knowledge, Copenhagen, Hovedstaden, DK, 27; Fussler, C., \& James, P. (1996). Driving eco-innovation: a breakthrough discipline for innovation and sustainability. London: Pitman Publishing; Nidumolu, R., Prahalad, C. K., \& Rangaswami, M. R. (2009). Why sustainability is now the key driver of innovation. Harvard Business Review, 87(9), 56-64; Sharma, S. (2000). Managerial interpretations and organizational context as predictors of corporate choice of environmental strategy. Academy of Management Journal, 43(4), 681-697. doi: 10.2307/1556361; Sharma, S., Pablo, A. L., \& Vredenburg, H. (1999). Corporate environmental responsiveness strategies: the importance of issue interpretation and organizational context. The Journal of Applied Behavioral Science, 35(1), 87-108. doi: 10.1177/0021886399351008; Sharma, S., Aragón-Correa, J. A., \& Rueda-Manzanares, A. (2007). The contingent influence of organizational capabilities on proactive environmental strategy in the service sector: an analysis of North American and European Ski Resorts. Canadian Journal of Administrative Sciences, 24(4), 268-283. doi: 10.1002/CJAS.35

Para comprovação ou não da composição desse construto no que se refere às variáveis, inicialmente definidas a partir da literatura, foi utilizada a técnica estatística multivariada de Análise Fatorial Exploratória (AFE). Os resultados foram satisfatórios, pois a medida da adequação da 
amostragem pelo teste de Kaiser-Meyer-Olkin (KMO), adequação da análise fatorial, resultou em 0,833, que é considerado um valor alto, indicando que a AFE é adequada (Cooper \& Schindler, 2011). Além disso, o teste de esfericidade de Bartlett apresentou o valor de 653,662, com significância de $p=0,000$, o que também demonstra a adequação da análise fatorial (Field, 2009).

A partir desses testes, verificou-se que os carregamentos dos fatores ficaram entre moderadamente importantes e acima de serem considerados muito importantes, exceto a Var09, que ficou com uma carga em valores aceitáveis, conforme os parâmetros de Hair et al. (2005). No caso da variância total explicada, ficou em 57\%, o que se justifica pela opção da determinação do número de fatores a priori (Hair et al., 2005). Ou seja, partiu-se do já definido pela literatura, que são os dois fatores (estratégias reativas e proativas), para verificar o comportamento das variáveis nesse construto. Enfim, a partir da AFE, pôde-se verificar que o construto das estratégias de ecoinovação resultou nos mesmos agrupamentos das variáveis dentro do construto, conforme definidos na revisão de literatura. Isto é, as variáveis foram confirmadas em relação ao que foi apresentado na literatura, compondo as mesmas dimensões.

Assim, com as hipóteses e esses construtos definidos, passou-se à análise dos dados, que serão apresentadas no tópico 4, iniciando com a caracterização da amostra.

\section{Caracterização da Amostra, Análises e Teste das Hipóteses}

A amostra deste estudo foi constituída por 117 empresas de todas as regiões do Brasil. A representatividade dessa amostra foi analisada no contexto regional brasileiro, relacionada com a participação das empresas na Pesquisa Industrial Anual (PIA-Empresa), do Instituto Brasileiro de Geografia e Estatística (IBGE, 2010a, 2010b). Para tanto, foi realizado o teste de adequação do ajuste do Quiquadrado (goodness of fit) com o intuito de testar se a frequência de empresas na amostra em cada área geográfica do Brasil é ou não similar à sua população (Maroco, 2003).

Com esse teste, foi possível avaliar a amostra de empresas utilizada neste estudo em relação à composição de empresas por região do Brasil, constantes da PIA-Empresa. O resultado indicou que a amostra é representativa $(\mathrm{p}=0,159)$, ou seja, é possível afirmar que a proporção de empresas de cada região na amostra é similar à proporção das empresas de cada região no total do Brasil. Isso permite inferir que a amostra é representativa para o Brasil como um todo e permite generalizar o estudo para o contexto nacional.

Quanto ao porte, o número significativo de empresas respondentes $(45 \%)$ pertence ao porte médio, conforme o critério do Serviço Brasileiro de Apoio às Micro e Pequenas Empresas (SEBRAE, 2012). Outra parcela significativa é de porte pequeno (34\%), o porte grande também aparece em números importantes (17\%), se considerado o setor de atividade, e as microempresas constituem a menor parcela da amostra (4\%).

Adicionalmente, a caracterização da amostra de empresas apresenta os seguintes aspectos: possui origem brasileira em sua maioria (97\%), com destaque para aquelas com capital controlador exclusivamente nacional (80\%); são organizações com tempo de existência na média de 36 anos; sendo que em $63 \%$ delas o principal mercado de atuação é o nacional.

As pessoas que se apresentaram como respondentes da pesquisa, em sua maioria, atuam em cargos de direção ou gerência (66\%) e trabalham na empresa há, aproximadamente, 10 anos.

Para a análise de regressão logística, o primeiro passo consistiu na caracterização das empresas da amostra em função das estratégias de ecoinovação adotadas, a partir da média dos escores nos construtos estratégias proativas e estratégias reativas. $\mathrm{Na}$ Tabela 2 foram apresentadas 13 variáveis selecionadas para identificar as estratégias ecoinovadoras das empresas, sendo que cinco delas identificam as estratégias reativas (Var05 a Var09) e as outras oito, as estratégias proativas (Var10 a 
Var17). Esses dois construtos (estratégias reativas e estratégias proativas) foram utilizados para identificar, dicotomicamente, o perfil das empresas (caracterização) que determinaria a variável dependente na regressão logística. Na Tabela 1, foram apresentadas quatro variáveis (Var01 a Var 04) referentes à questão da regulamentação ambiental. Essas variáveis foram utilizadas como variáveis independentes na regressão logística.

Com base nos escores individuais em cada construto, as empresas foram divididas em dois grupos pela utilização da técnica de agrupamento (cluster). De acordo com Malhotra (2006), a análise de cluster é utilizada quando "não há qualquer informação a priori sobre a composição do grupo ou cluster para qualquer um de seus objetos. Os grupos ou clusters são sugeridos pelos dados, e não a priori" (p. 573) (grifos no original). O tipo de método de cluster utilizado neste estudo foi o hierárquico aglomerativo, método de variância Ward. Inicialmente, foi realizado esse teste para verificação do número recomendado de clusters, sendo visualizados dois grupos a partir do resultado apresentado pelo gráfico de dendrograma. Para permitir a identificação de cada grupo e sua caracterização, foi construído o gráfico de error bars, para as médias dos escores de cada construto em cada grupo, conforme pode ser visualizado na Figura 1, onde foram inseridas as taxonomias criadas para cada grupo.

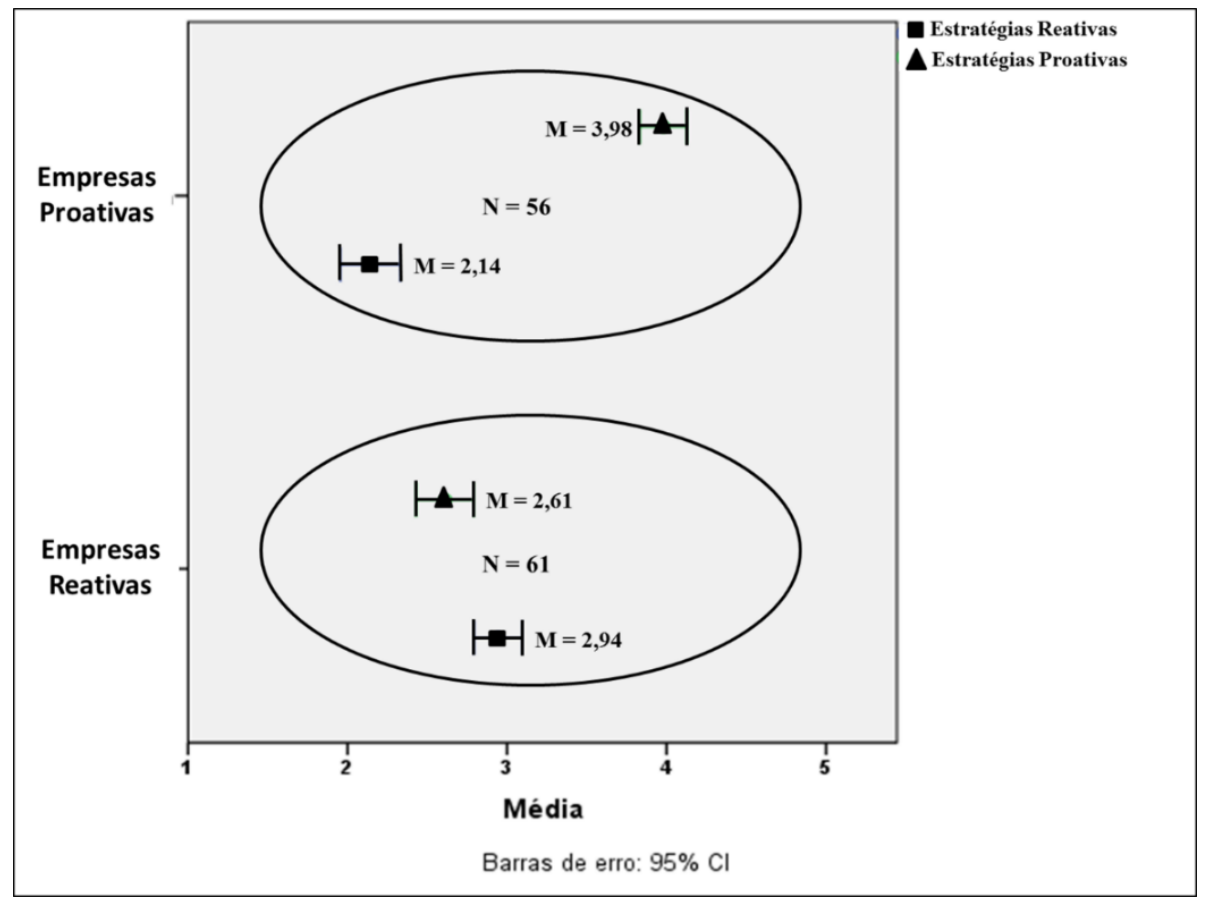

Figura 1. Agrupamento das Empresas

A partir do gráfico apresentado na Figura 1, pode-se vislumbrar uma taxonomia das empresas participantes do estudo conforme os seus agrupamentos. No cluster 1, aparecem as empresas proativas (56 empresas $-48 \%$ ), que são aquelas com escores maiores para as estratégias proativas $(\mathrm{M}=3,98)$ e menores para as reativas $(\mathrm{M}=2,14)$. $\mathrm{O}$ teste $t$ para amostras independentes indica que as médias dos escores das estratégias são estatisticamente diferentes (valor $\mathrm{p}=0,000$ ). No entender da análise realizada neste estudo, neste cluster, já se percebe a gestão ambiental como uma estratégia corporativa e, por isso, essas empresas são as consideradas como possíveis ecoinovadoras, que enfatizam o desenvolvimento sustentável, resultando na redução de riscos ambientais, poluição e outros impactos negativos da utilização dos recursos naturais. E podem ser associadas às empresas que Aragón-Correa (1998) e Sharma et al. (1999) consideram como aquelas que desenvolvem ações voluntárias de prevenção dos impactos ambientais, criando vantagem competitiva por meio da adoção de tecnologias ecoinovadoras.

No cluster 2, aparecem as empresas reativas (61 empresas - 52\%), que são aquelas com escores mais altos para as estratégias reativas $(\mathrm{M}=2,94)$ e menores para as proativas $(\mathrm{M}=2,61)$. O teste $t$ para amostras independentes indica que as médias dos escores das estratégias são estatisticamente diferentes (valor $\mathrm{p}=0,000$ ). Nelas, a gestão ambiental não aparenta ser uma estratégia organizacional. Elas podem 
ser consideradas as empresas que Sharma (2000) e Sharma et al. (1999) definem como aquelas que desenvolvem (apenas) as ações impostas externamente pela legislação ambiental, por meio do controle da poluição com tecnologias corretivas, em que o envolvimento da alta administração é esporádico.

Na sequência, foi realizada a regressão logística tendo como variável dependente o perfil ecoinovador das empresas, determinado pela caracterização como proativas, aquelas empresas pertencentes ao cluster 1 , e, como reativas, as pertencentes ao cluster 2.

Para a variável dependente, dicotômica, as empresas classificadas como as que adotam estratégias proativas foram identificadas com o algarismo 1 (um) e as empresas classificadas como aquelas que adotam estratégias reativas foram identificadas com o algarismo 0 (zero). Para as variáveis independentes, consideraram-se as quatro variáveis (conforme descritas na Tabela 1), que compõem o construto regulamentação. A equação geral da regressão logística pode ser escrita como:

$$
Y=b_{0}+b_{1} X_{1}+b_{2} X_{2}+b_{3} X_{3}+b_{4} X_{4}+\epsilon
$$

Onde:

$$
\begin{aligned}
& Y=\text { Estratégias de ecoinovação } \\
& X_{\mathrm{n}}=\text { Variáveis da regulamentação }
\end{aligned}
$$

$\mathrm{Na}$ primeira versão da equação, as variáveis $\mathrm{X}_{2}$ (Var02) e $\mathrm{X}_{3}$ (Var03) não se mostraram estatisticamente significantes e foram retiradas do modelo de regressão logística. Assim, a equação geral do modelo final foi escrita como:

$$
Y=b_{0}+b_{1} X_{1}+b_{4} X_{4}+\epsilon
$$

Na Tabela 3 é apresentado o modelo de regressão logística onde estão indicados os coeficientes

\begin{tabular}{|c|c|c|c|c|c|c|}
\hline & \multicolumn{3}{|c|}{ Modelo 1} & \multicolumn{3}{|c|}{ Modelo 2} \\
\hline & $b$ & p value & $\operatorname{Exp}(B)$ & $b$ & p value & $\operatorname{Exp}(B)$ \\
\hline Constante & $-4,882$ & 0,000 & 0,008 & $-5,173$ & 0,000 & 0,006 \\
\hline Var01 & 0,987 & 0,001 & 2,683 & 0,885 & 0,001 & 2,424 \\
\hline Var02 & $-0,077$ & 0,729 & 0,926 & - & - & - \\
\hline Var03 & $-0,197$ & 0,552 & 0,821 & - & - & - \\
\hline Var04 & 0,627 & 0,026 & 1,873 & 0,539 & 0,034 & 1,714 \\
\hline $\mathbf{R}^{2}$ de Nagelkerke & \multicolumn{3}{|c|}{0,390} & \multicolumn{3}{|c|}{0,385} \\
\hline $\mathbf{R}^{2}$ Cox e Snell & \multicolumn{3}{|c|}{0,293} & \multicolumn{3}{|c|}{0,289} \\
\hline Hosmer e Lemeshow & \multicolumn{3}{|c|}{$12,138(\mathrm{p}=0,145)$} & \multicolumn{3}{|c|}{$4,539(\mathrm{p}=0,604)$} \\
\hline
\end{tabular}
da função logística para a regulamentação.

Tabela 3

\section{Regressão Logística para a Regulamentação}

Hair, Black, Babin, Anderson e Tatham (2009) apresentam três abordagens para avaliar o ajuste geral do modelo. A primeira delas é composta por duas medidas. "O teste qui-quadrado para a variação no valor -2LL do modelo base, que é compatível com o teste $F$ geral em regressão múltipla" (Hair et al., 2009, p. 295). Neste caso, o -2 logaritmo de verossimilhança (-2LL) foi de 122,153, significante ao nível de 0,000 . A segunda medida é "o teste de Hosmer e Lemeshow que mostra a significância do modelo logístico de uma variável" (Hair et al., 2009, p. 295). O resultado do teste apresenta: Quiquadrado $=4,539 ; \mathrm{df}=6 ; \mathrm{p}=0,604$, indicando um ajuste aceitável. 
A segunda abordagem é composta por medidas que são compatíveis com o $\mathrm{R}^{2}$ em modelos de regressão múltipla. Trata-se do $\mathrm{R}^{2}$ de Cox e Snell $(0,289)$ e do $\mathrm{R}^{2}$ de Nagelkerke $(0,385)$. Ambos tiveram resultados relativamente baixos, indicando que, segundo o $\mathrm{R}^{2}$ de Nagelkerbe, o modelo é capaz de explicar 38,5\% das variações registradas na variável dependente (propensão estratégica).

A terceira abordagem é a identificação da precisão de classificação. A acurácia global de classificação é de $71,8 \%$. Ou seja, é a capacidade que o modelo tem de inclusão correta das empresas em um ou outro grupo.

Segundo os três tipos básicos de medidas de ajuste geral, o modelo proposto apresenta níveis adequados de significância estatística e prática.

O primeiro aspecto relevante a ser considerado é o direcionamento da relação, representado pelo sinal (positivo ou negativo) das variáveis da regulamentação. Nos dados da equação, percebe-se que a regulamentação tem associação positiva com as estratégias de ecoinovação (reativas e proativas). Os resultados acompanham o que foi identificado na literatura, ou seja, que a regulamentação ambiental está associada à determinação das estratégias. Porém, em contraponto a esta no que tange às organizações categorizadas como aquelas que adotam estratégias ecoinovadoras reativas, pois a literatura indica que, no caso destas organizações, a associação entre a regulamentação e as estratégias seria mais forte (Buysse \& Verbeke, 2003; Nidumolu, Prahalad, \& Rangaswami, 2009; Sharma et al., 1999).

Constatou-se ainda que, a partir dos cálculos propostos por Field (2009, p. 239), no que se referee ao $\operatorname{Exp}(\mathrm{B})$, as chances de as empresas perceberem a regulamentação, sob a perspectiva de custo (Var01), como fator relevante para as estratégias é 2,424 vezes maior para as organizações que adotam estratégias proativas em relação às organizações que adotam estratégias reativas. Assim, também, as chances de as empresas perceberem a regulamentação, sob a perspectiva de oportunidade (Var04), como fator relevante às estratégias, é 1,714 vezes maior para as organizações que adotam estratégias proativas em relação às organizações que adotam estratégias reativas. Em outras palavras, existe 4,154 vezes mais chance de uma empresa que adota estratégia proativa perceber a importância da regulamentação do que empresas que adotam estratégias reativas.

Este resultado pode indicar que as organizações que adotam estratégias proativas atribuem maior importância à regulamentação ambiental porque buscam se antecipar a elas, enquanto que as organizações que adotam estratégias reativas não atribuem tanta importância à regulamentação porque simplesmente buscam se adaptar às exigências. Este resultado corrobora o mencionado nos estudos de Ansanelli (2011), Ashford (2000), Corazza (2003b) e de Porter e Linde (1995, 1999).

\section{Considerações Finais}

Este estudo surgiu da verificação de que na literatura há falta de pesquisas que tratem das estratégias de ecoinovação, seus condutores e efeitos. Por isso, teve por objetivo analisar a associação da regulamentação ambiental com a adoção de estratégias de ecoinovação proativas e reativas no setor brasileiro de celulose, papel e produtos de papel. Em função da literatura, foram levantadas duas hipóteses, desdobradas em uma hipótese nula e uma alternativa para cada uma delas.

Para a análise de regressão logística, foi inicialmente realizada a caracterização das empresas de acordo com as estratégias de ecoinovação adotadas. Para tanto, foi utilizada a análise de cluster, em que as empresas da amostra foram definidas como proativas (56 empresas - 48\%) e reativas (61 empresas $52 \%)$.

A partir da análise de regressão logística, puderam ser inferidos alguns resultados que incrementam a teoria existente. Os coeficientes beta (B) da equação indicam uma associação positiva da regulamentação com as estratégias de ecoinovação. Entretanto o valor $\operatorname{Exp(B)~conjunto~indica~que~}$ esta associação é 4,154 vezes maior no caso das empresas categorizadas como adotantes de estratégias 
proativas em relação às organizações categorizadas como adotantes das estratégias reativas. Os resultados mostram que o teste das hipóteses nulas $\left(\mathrm{H}_{0} \mathrm{~A}\right.$ e $\left.\mathrm{H}_{0} \mathrm{~B}\right)$ falhou em rejeitá-las, confirmando a associação entre a regulamentação e as estratégias de ecoinovação. Mas as organizações participantes deste estudo consideram a regulamentação ambiental como uma oportunidade estratégica, resultando em ações proativas de maneira mais contundente que em ações reativas.

Nesse sentido, os achados neste estudo não corroboram a teoria existente, a qual considera que as empresas acreditam numa relação de aumento de custos, perda de produtividade e sem benefícios a curto prazo, resultante da incidência da regulamentação ambiental (Barbieri, 2011; Blackburn, 2008; Romeiro \& Salles, 1996). Além disso, esse resultado se contrapôs à ideia de que a regulamentação é vista, pelas empresas, como pressões de órgãos reguladores, obstáculos jurídico-legais, que demandam grandes investimentos sem possibilidade de recuperação (Barbieri, 2011; Donaire, 2007; Young et al., 2009).

O resultado, todavia, insere-se na perspectiva evolucionista, a qual enfatiza que as empresas tendem a ver a restrição ambiental cada vez mais como oportunidades tecnológicas e vantagens competitivas (Romeiro \& Salles, 1996). Também coaduna com a visão teórica de que a regulamentação deve orientar a empresa a inovar, e a empresa deve ver esta pressão como melhoria de produtividade para a competitividade (Ansanelli, 2011; Ashford, 2000; Corazza, 2003b; Porter \& Linde, 1995).

Uma síntese dos resultados das análises das hipóteses pode ser visualizada na Tabela 4, com as concepções dos estudos anteriores e os achados desta pesquisa.

Tabela 4

\section{Síntese dos Resultados do Teste das Hipóteses}

Resultados

Hipóteses e base teórica . $\mathbf{H}_{0} \mathbf{A}$ - A percepção dos executivos sobre a regulamentação ambiental é que ela está associada positivamente à adoção de estratégias ecoinovadoras reativas (Barbieri, 2011; Blackburn, 2008; Donaire, 2007; Romeiro \& Salles, 1996; Young et al., 2009).

. H1A - A percepção dos executivos sobre a regulamentação ambiental é que ela está associada negativamente à adoção de estratégias de ecoinovação reativas.

HoB - A percepção dos executivos sobre a regulamentação ambiental é que ela está associada positivamente à adoção de estratégias de ecoinovação proativas (Ansanelli, 2011; Ashford, 2000; Corazza, 2003b; Porter \& Linde, 1995, 1999).

. H1B - A percepção dos executivos sobre a regulamentação ambiental é que ela está associada negativamente à adoção de estratégias de ecoinovação proativas.

Resultado O teste das hipóteses falhou $\mathrm{O}$ beta (B) da equação indica associação positiva da em rejeitar as hipóteses nulas regulamentação e das estratégias de ecoinovação, no $\left(\mathrm{H}_{0} \mathrm{~A}\right.$ e $\left.\mathrm{H}_{0} \mathrm{~B}\right) \quad$ entanto o valor $\operatorname{Exp}(\mathrm{B})$ conjunto indica que esta associação é 4,154 vezes maior no caso das empresas categorizadas como adotantes de estratégias proativas em relação às organizações categorizadas como adotantes das estratégias reativas.

Concepção verificada . Nas organizações participantes deste estudo, a regulamentação ambiental está neste estudo e aderência associada positivamente com as estratégias de ecoinovação proativas, em termos a estudos anteriores mais contundentes que com as estratégias de ecoinovação reativas.

. Este resultado se insere na perspectiva evolucionista, a qual postula que as empresas veem a regulamentação ambiental menos como custo e mais como oportunidade (Romeiro \& Salles, 1996), orientando-as para melhoria de produtividade e de competitividade (Ansanelli, 2011; Ashford, 2000; Corazza, 2003b; Porter \& Linde, 1995). 
Assim, pode-se considerar que este estudo teve como contribuição importante para o avanço do conhecimento na área de estratégias de ecoinovação a definição e análise das variáveis dos construtos da pesquisa. Mais especificamente, fatores da regulamentação ambiental que influenciam na adoção de estratégias de ecoinovação reativas e proativas, trazendo incrementos à literatura existente. Ressalta-se que a originalidade está no fato de ser um estudo especialmente concebido e aplicado de forma empírica a partir de análises sobre a gestão da ecoinovação, quanto aos condutores e efeitos, permitindo o estudo aprofundado, o qual é considerado uma lacuna na literatura.

Portanto este estudo contribui tanto para a teoria existente como para a gestão de ecoinovações nas organizações. Nesse sentido, foi estabelecido um quadro de referência para a definição de metas de longo prazo nas políticas empresariais, apresentando os fatores e suas incidências nas estratégias de ecoinovação das empresas, também, permitindo análises setoriais. Com essas contribuições, este estudo pode servir de guia na condução do enfoque inovativo da gestão ambiental em indústrias do setor e de outros setores de atividade, bem como no incremento de pesquisas nessa área.

Por fim, há de se destacar que as limitações da pesquisa estão relacionadas aos surveys por amostragem, pelos quais raramente é possível determinar o grau de precisão dos achados, bem como em razão de os questionários terem sido respondidos por apenas uma pessoa de cada empresa, não tendo controle da intencionalidade e da forma de resposta. Entretanto, para garantir aspectos de validade e confiabilidade, foi realizada validade de conteúdo e pré-teste do questionário, além de serem tomadas outras precauções em relação à metodologia adotada.

Como pesquisas futuras, este estudo aponta algumas áreas que se evidenciam como importantes linhas de pesquisa para o levantamento das questões ainda inexploradas sobre a ecoinovação. Esses temas podem ser relacionados com a análise dos impactos da adoção de estratégias de ecoinovação no desempenho social, ambiental e econômico das empresas; o ecoempreendedorismo, ou seja, a criação de empresas de base tecnológica e na linha ecológica; a análise das fontes de informação e relações de transferência de tecnologia no contexto da ecoinovação; a definição de políticas governamentais, para tanto, levantando os estilos de política ambiental propícios à inovação, estudos comparativos de efeitos e regimes de políticas sobre a inovação ambiental; e uma análise empírica internacional sobre as características específicas da ecoinovação em diferentes sistemas nacionais e a capacidade inovativa ambiental de diferentes países.

\section{Agradecimentos}

Agradecemos à equipe da RAC e aos pareceristas anônimos, que muito contribuíram para a qualidade deste estudo. E também ao CNPQ, pelo auxílio financeiro à execução desta pesquisa.

\section{Referências}

Andersen, M. M. (2010, June). On the faces and phases of eco-innovation - on the dynamics of the greening of the economy. Proceedings of the DRUID Conference - Opening up Innovation: Strategy, Organization and Technology, London, Greater London, UK, 29.

Andrade, T. de (2004). Inovação tecnológica e meio ambiente: a construção de novos enfoques. Ambiente \& Sociedade, 7(1), 89-106. Recuperado de http://www.scielo.br/pdf/asoc/v7n1/23538.pdf. doi: 10.1590/S1414-753X2004000100006

Ansanelli, S. L. M. (2011). Exigências ambientais europeias: novos desafios competitivos para o complexo eletrônico brasileiro. Revista Brasileira de Inovação, 10(1), 129-160. 
Aragón-Correa, J. A. (1998). Strategic proactivity and firm approach to the natural environment. Academy of Management Journal, 41(5), 556-567. doi: 10.2307/256942

Ashford, N. A. (2000). An innovation-based strategy for a sustainable environment. In J. Hemmelskamp, K. Rennings, \& F. Leone (Eds.), Innovation-oriented environmental regulation: theoretical approach and empirical analysis ZEW Economic Studies (pp. 67-107). New York: $\begin{array}{llll}\text { Springer } & \text { Verlag, } & \text { Heidelberg. } & \text { Retrieved }\end{array}$ http://18.7.29.232/bitstream/handle/1721.1/1590/Potsdam.pdf?sequence=1

Barbieri, J. C. (2011). Gestão ambiental empresarial: conceitos, modelos e instrumentos (3a ed.) São Paulo: Saraiva.

Beuren, I. M., \& Oro, I. M. (2014). Relação entre estratégia de diferenciação e inovação, e sistemas de controle gerencial. Revista de Administração Contemporânea, 18(3), 285-310. Recuperado de http://www.scielo.br/pdf/rac/v18n3/v18n3a04.pdf. doi: 10.1590/1982-7849rac20141394

Blackburn, W. R. (2008). The sustainability handbook. Washington: Environmental Law Institute.

Buysse, K., \& Verbeke, A. (2003). Proactive environmental strategies: a stakeholder management perspective. Strategic Management Journal, 24(5), 453-470. doi: 10.1002/smj.299

Caracuel, J. A., Torres, M. Á. E., Torres, N. E. H., \& Salazar, M. D. V. (2011). La influencia de la diversificación y experiencia internacional en la estrategia medioambiental proactiva de las empresas. Investigaciones Europeas de Direccion y Economia de la Empresa, 17(1), 75-91. doi: $10.1016 / \mathrm{S} 1135-2523(12) 60045-8$

Cooper, D. R., \& Schindler, P. S. (2011). Métodos de pesquisa em administração (10a ed.). Porto Alegre: Bookman.

Corazza, R. I. (2003a). Economia, tecnologia e meio ambiente: comentários sobre aspectos positivos e normativos da economia do meio ambiente. Ensaios FEE, 24(2), 479-498.

Corazza, R. I. (2003b). Gestão ambiental e mudanças da estrutura organizacional. RAE-Eletrônica, 2(2), 1-23. Recuperado de http://www.scielo.br/pdf/raeel/v2n2/v2n2a06.pdf. doi: 10.1590/S167656482003000200006

Costa, S., Borini, F. M., \& Amatuci, M. (2013). Inovação global de subsidiárias estrangeiras localizadas em mercados emergentes. Revista de Administração Contemporânea, 17(4), 459-478. Recuperado de http://www.scielo.br/pdf/rac/v17n4/a05v17n4.pdf. doi: 10.1590/S141565552013000400005

Donaire, D. (2007). Gestão ambiental na empresa (2a ed.). São Paulo: Atlas.

Field, A. (2009). Descobrindo a estatística usando o SPSS (2a ed.). Porto Alegre: Artmed.

Foray, D., \& Grübler, A. (1996). Technology and the environment: an overview. Technological Forecasting and Social Change, 53(1), 3-13. doi: 10.1016/0040-1625(95)00064-X

Foxon, T., \& Andersen, M. M. (2009, June). The greening of innovation systems for eco-innovation towards an evolutionary climate mitigation policy. Proceedings of the DRUID Summer Conference - Innovation, Strategy and Knowledge, Copenhagen. Hovedstaden, DK, 27.

Freeman, C. (1996). The greening of technology and models of innovation. Technological forecasting and social change, 53(1), 27-39. doi: 10.1016/0040-1625(96)00060-1

Fussler, C., \& James, P. (1996). Driving eco-innovation: a breakthrough discipline for innovation and sustainability. London: Pitman Publishing. 
Hair, J. F., Jr., Babin, B., Money, A. H., \& Samouel, P. (2005). Fundamentos de métodos de pesquisa em administração. Porto Alegre: Bookman.

Hair, J. F., Jr., Black, W. C., Babin, B., Anderson, R. E., \& Tatham, R. L. (2009). Análise multivariada de dados (6a ed.). Porto Alegre: Bookman.

Hart, S. (1995). A natural-resource-based view of the firm. The Academy of Management Review, 20(4), 986-1014. doi: 10.5465/AMR.1995.9512280033

Instituto Brasileiro de Geografia e Estatística. (2010a). Tabela 2.5 - Dados gerais das unidades locais industriais de empresas industriais com 5 ou mais pessoas ocupadas, segundo as divisões e os grupos de atividades - Brasil - 2010. Recuperado de $\mathrm{ftp} / / / \mathrm{ftp}$. ibge.gov.br/Industrias_Extrativas_e_de_Transformacao/Pesquisa_Industrial_Anual/Em presa2010/tabela2_5.pdf

Instituto Brasileiro de Geografia e Estatística. (2010b). Tabela 2.10 - Dados gerais das unidades locais industriais de empresas industriais com 5 ou mais pessoas ocupadas, por Unidades da Federação, segundo as divisões de atividades - Região Centro-Oeste - 2010. Recuperado de $\mathrm{ftp} / / / \mathrm{ftp}$. ibge.gov.br/Industrias_Extrativas_e_de_Transformacao/Pesquisa_Industrial_Anual/Em presa2010/tabela2_10.pdf

Kuhl, M. R., \& Cunha, J. C. (2013). Obstáculos à implementação de inovações no Brasil. Brazilian Business Review, 10(2), 1-25.

Lei $n$. 6.938, de 31 de agosto de 1981. (1981). Dispõe sobre a política nacional do meio ambiente, seus fins e mecanismos de formulação e aplicação, e dá outras providências. Recuperado de https://www.planalto.gov.br/ccivil_03/leis/16938.htm

Maçaneiro, M. B., Cunha, S. K. da, \& Balbinot, Z. (2013). Drivers of the adoption of eco-innovations in the pulp, paper, and paper products industry in Brazil. Latin American Business Review, 14(34), 179-208. doi: 10.1080/10978526.2013.833465

Malhotra, N. K. (2006). Pesquisa de marketing: uma orientação aplicada (4a ed.). Porto Alegre: Bookman.

Maroco, J. (2003). Análise estatística com utilização do SPSS (2a ed.). Lisboa: Sílabo.

Menguc, B., Auh, S., \& Ozanne, L. (2010). The interactive effect of internal and external factors on a proactive environmental strategy and its influence on a firm's performance. Journal of Business Ethics, 94(2), 279-298. doi: 10.1007/s10551-009-0264-0

Motta, R. S. da, \& Young, C. E. F. (1997). Introdução. In R. S. da Motta \& C. E. F. Young (Coords.), Projeto Instrumentos econômicos para a gestão ambiental (Relatório Final, pp. 5-7). Rio de Janeiro. Recuperado de http://www.ie.ufrj.br/gema/pdfs/projeto.pdf

Nidumolu, R., Prahalad, C. K., \& Rangaswami, M. R. (2009). Why sustainability is now the key driver of innovation. Harvard Business Review, 87(9), 56-64.

Porter, M. E., \& Linde, C. van der (1995). Toward a new conception of the environmentcompetitiveness relationship. The Journal of Economic Perspective, 9(4), 97-118. doi: 10.1257/jep.9.4.97

Porter, M. E., \& Linde, C. van der (1999). Verde e competitivo: acabando com o impasse. In M. E. Porter (Org.), Competição = on competition: estratégias competitivas essenciais (pp. 371-397). Rio de Janeiro: Campus. 
Rennings, K. (2000). Redefining innovation - eco-innovation research and the contribution from ecological economics. Ecological Economics, 32(2), 319-332. doi: 10.1016/S09218009(99)00112-3

Ribeiro, F. F., Oliveira, M. M., Jr., \& Borini, F. M. (2012). Internacionalização acelerada de empresas de base tecnológica: o caso das Born Globals Brasileiras. Revista de Administração Contemporânea, 16(6), 866-888. Recuperado de http://www.scielo.br/pdf/rac/v16n6/a07v16n6.pdf. doi: 10.1590/S1415-65552012000600007

Romeiro, A. R., \& Salles, S., Filho (1996). Dinâmica de inovações sob restrição ambiental. In A. R. Romeiro, B. P. Reydon, \& M. L. A. Leonardi (Orgs.), Economia do meio ambiente: teoria, políticas e a gestão de espaços regionais (pp. 83-122). Campinas: UNICAMP.

Schmidheiny, S. (1992). Mudando o rumo: uma perspectiva empresarial global sobre desenvolvimento e meio ambiente. Rio de Janeiro: Ed. da FGV.

Serviço Brasileiro de Apoio às Micro e Pequenas Empresas. (2012). Critérios e conceitos para classificação de empresas. Recuperado de http://arquivopdf.sebrae.com.br/uf/goias/indicadoresdas-mpe/classificacao-empresarial/integra_bia/ident_unico/97

Sharma, S. (2000). Managerial interpretations and organizational context as predictors of corporate choice of environmental strategy. Academy of Management Journal, 43(4), 681-697. doi: $10.2307 / 1556361$

Sharma, S., Aragón-Correa, J. A., \& Rueda-Manzanares, A. (2007). The contingent influence of organizational capabilities on proactive environmental strategy in the service sector: an analysis of North American and European Ski Resorts. Canadian Journal of Administrative Sciences, 24(4), 268-283. doi: 10.1002/CJAS.35

Sharma, S., Pablo, A. L., \& Vredenburg, H. (1999). Corporate environmental responsiveness strategies: the importance of issue interpretation and organizational context. The Journal of Applied Behavioral Science, 35(1), 87-108. doi: 10.1177/0021886399351008

Young, C. E. F., Podcameni, M. G. B., Mac-Knight, V., \& Oliveira, A. S. (2009). Determinants of environmental innovation in the Brazilian industry. Proceedings of the Congreso de la Asociación Latinoamericana y del Caribe de Economistas Ambientales y de Recursos Naturales, Heredia, Costa Rica, $4 . \quad$ Retrieved from http://www.ie.ufrj.br/gema/pdfs/DETERMINANTS\%20OF\%20ENVIRONMENTAL\%20INN OVATION\%20IN\%20THE\%20BRAZILIAN\%20INDUSTRY.pdf

\section{Dados dos Autores}

Marlete Beatriz Maçaneiro

Rua Padre Salvador, 875, Santa Cruz, 85015-430, Guarapuava, PR, Brasil. E-mail: marlete.beatriz@yahoo.com.br

Sieglinde Kindl da Cunha

Rua Prof. Pedro Viriato Parigot de Souza, 5300, 81280-330, Curitiba, PR, Brasil. E-mail: skcunha21@gmail.com

Marcos Roberto Kuhl

Rua Padre Salvador, 875, Santa Cruz, 85015-430, Guarapuava, PR, Brasil. E-mail: marcosrobertokuhl@ yahoo.com.br

João Carlos da Cunha

Rua Prof. Pedro Viriato Parigot de Souza, 5300, 81280-330, Curitiba, PR, Brasil. E-mail: jccpositivo@ gmail.com 